\title{
基于划刻实验的单晶锗材料去除机理研究
}

\author{
耿瑞文 ${ }^{1}$, 杨晓京 ${ }^{1}$, 谢启明 ${ }^{2}$, 李 芮 $^{3}$, 罗 良 ${ }^{1}$
}

(1. 昆明理工大学 机电工程学院, 昆明 $650500 ; 2$. 昆明物理研究所, 昆明 $650233 ; 3$. 昆明理工大学 环境科学与 工程学院, 昆明 650500)

摘 要: 采用 Cube 压头对单晶锗进行变载与恒载纳米划刻实验, 利用扫描电子显微镜和原子力显微镜对已加工表 面进行观测, 根据表面形貌将划刻过程分为延性域、脆塑转变域及脆性域三种, 对各个阶段的表面成型及材料去除 方式进行了研究。使用最小二乘法对不同阶段划刻力进行非线性拟合, 并利用相关系数检验拟合函数可靠性, 结果 表明划刻力与划刻深度存在强相关性。同时分析了单晶锗的弹性回复率随划刻距离的变化趋势, 结果表明工件的弹 性回复率将从纯弹性阶段的 1 逐步回落至 0.76 左右。基于脆塑转变临界载荷, 以裂纹萌生位置作为脆塑转变标志, 首次结合工件已加工表面弹性回复, 提出一种适用于计算单晶锗的脆塑转变临界深度模型, 其脆塑转变临界深度 为 $489 \mathrm{~nm}$ 。

关 键 词: 单晶锗; 纳米划刻实验; 表面形貌; 材料去除; 弹性回复; 脆塑转变

中图分类号: TN304 文献标识码: A

\section{Material Removal Mechanism of Monocrystalline Germanium Based on Nano-scratch Experiment}

\author{
GENG Rui-Wen ${ }^{1}$, YANG Xiao-Jing ${ }^{1}$, XIE Qi-Ming ${ }^{2}$, LI Rui ${ }^{3}$, LUO Liang ${ }^{1}$
}

(1. Faculty of Mechanical and Electrical Engineering, Kunming University of Science and Technology, Kunming 650500, China; 2. Kunming Institute of Physics, Kunming 650233, China; 3. Faculty of Environmental Science and Engineering, Kunming University of Science and Technology, Kunming 650500, China)

\begin{abstract}
A varied and constant load nano-scratch experiment on monocrystalline germanium was performed by Cube indenter. The surface morphology of the groove was observed by scanning electron microscope (SEM) and atomic force microscope (AFM), and the scratch process was divided into three regimes, that is ductile regime, ductile-brittle transition regime and brittle regime based on observations. The material removal mechanism of different regimes was also analyzed. The least square method was used to establish nonlinear curve fittings between scratch depth and scratch force, and the correlation coefficient was used to verify reliability of fitting functions. Result shows that there is a strong correlation between scratch depth and scratch force. Meanwhile, the tendency of elastic recovery rate with scratch distance was analyzed. As the result shows, the elastic recovery rate gradually decreases from 1 in the pure elastic stage to about 0.76 in stable stage. Based on ductile-brittle translation critical load, a model is proposed to calculate the critical depth where the elastic recovery rate is firstly considered, and the ductile-brittle translation critical depth for monocrystalline germanium is $489 \mathrm{~nm}$.
\end{abstract}

Key words: monocrystalline germanium; nano-scratch experiment; surface morphology; material removal; elastic recovery; ductile-brittle translation

收稿日期：2018-10-18; 收到修改稿日期：2018-11-26

基金项目: 国家自然科学基金(51765027) National Natural Science Foundation of China (51765027)

作者简介: 耿瑞文(1993-), 男, 博士研究生. E-mail: precious.grw@gmail.com

通讯作者: 杨晓京, 教授. E-mail: xjyang@vip.sina.com 
单晶锗是一种重要的半导体材料，在微纳制造、 电子、光学等领域有着广泛的应用, 常用作半导体器 件、红外光学器件、太阳能电池祄底等材料 ${ }^{[1-2]}$ 。同 时单晶锗也是一种典型的硬脆性材料, 具有高强 度、高硬度、低断裂韧性等特点。为了克服硬脆性 材料的固有属性造成的加工困难, 研究者借助激 光、超声震动、高能离子束等手段开展辅助加工实

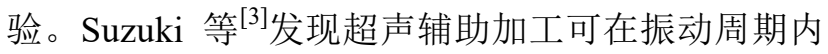
减小瞬时未切屑厚度和切削力, 进而改善单晶锗工 件表面质量。Wang 等 ${ }^{[4]}$ 对单晶锗进行离子注入处理, 发现处理后工件表面产生了均匀的非晶层, 其脆塑 转变临界深度较未改性单晶锗显著提高。Ravindra 等 ${ }^{[5]}$ 发现在相同载荷下激光辅助加工可在提高切削 深度的同时减小切削力, 从而提高刀具使用寿命。

尽管研究者针对硬脆性材料提出了多种辅助加 工措施, 但其表面成型特征与材料去除方式仍不清 晰。目前, 研磨和抛光是硬脆性材料传统加工手段, 却不适用于非球面光学元器件, 而单点金刚石车削 可实现复杂面型高次非球面工件的纳米级切削, 被 广泛地应用于硬脆性材料的超精密加工中。单晶锗 在实际加工中常面临崩碎、凹坑、白斑和麻点等缺 陷, 严重制约光学元件的生产效率并大幅提升生产 成本, 限制红外光学元器件的大规模应用。为了获 得光洁而完整的工件表面, 有必要研究单晶锗材料 去除机理。纳米划刻实验不仅可测量材料的杨氏模 量、硬度、摩擦系数等参数, 同时结合 SEM 和 AFM 可研究划刻过程中材料表面微观特性, 是一种研究 材料去除机理的常用手段 ${ }^{[6]}$ 。

为了探明单晶锗材料纳米去除机理, 本文对单 晶锗切片进行变载荷与定载荷划刻, 并根据其表面 的裂纹分布情况, 将划刻过程分为延性域(Ductile regime)、脆塑转变域(Ductile-brittle transition regime) 与脆性域(Brittle regime)三种。对于单晶锗划刻过程 中划痕的表面形貌、力学参数、材料去除方式进行 了分析与研究。首次结合已加工表面的弹性回复, 提出单晶锗脆塑转变临界深度计算模型, 对于指导 单晶锗光学元器件的生产加工具有重要意义。

\section{1 实验方法}

本研究采用 MTI 科晶公司生产的单晶锗切片, 通过单面化学机械抛光法处理, 其表面粗粘度 (RMS) 小于 $0.5 \mathrm{~nm}$, 工件尺寸为 $10 \mathrm{~mm} \times 10 \mathrm{~mm} \times$ $0.45 \mathrm{~mm}$ 。选取抛光后的(100)晶面作为实验面, 切片 在 1000 级超净工作室制备, 实验前将切片在丙酮中 浸泡 $10 \mathrm{~min}$, 在光学显微镜下观测其表面光洁。
纳米划刻实验在 Nano indenter G200 上进行, 采用 Scratch with lateral force 系统测量划刻过程中 的切向力与法向力, 选用压头形状可以在小范围保 持自相关 Cube 压头, 压头尖端曲率半径约为 $20 \mathrm{~nm}$, 各棱边间相互垂直。实验采用棱朝前方式划刻, 产 生的切屑将均匀分布于沟槽两侧且压头对切屑流动 的阻碍作用相较于其他划刻方式最小，划刻过程平 稳, 实验参数如表 1 。在划刻前后对工件表面高度进 行预扫描与后扫描，结合高分辨率的 Fei Quanta 400 场发射电子显微镜, 对划痕的整体及其局部断裂形 貌进行观测。使用 Bruker dimension icon 原子力显 微镜对划刻后划痕表面的三维形貌进行检测，以获 取划痕表面微观细节, 划刻实验示意图如图 1。

\section{2 结果与讨论}

\section{1 单晶锗变载荷划刻表面特征}

如图 2(a)为单晶锗沟槽整体表面形貌。工件首 先会经历完全弹性变形, 表现为在压头划过工件表 面后, 划痕会回弹至初始状态, 不产生材料去除, 因此工件的表面形貌不会发生改变。

随着压头载荷的增加, 单晶锗的划刻模式将进 入塑性变形阶段, 如图 2(b)。塑性变形分为耕犁与 切削两种模式 ${ }^{[7]}$ : 工件处于耕犁模式时, 由于材料 的塑性流动会在划痕的两侧产生隆起(Pile-up), 两 侧的隆起高度与划刻深度呈正相关。在切削模式中, 由于切屑的形成, 单晶锗材料将开始稳定去除, 造 成沟槽外隆起高度相对于耕犁模式有所减少。

表 1 纳米划刻实验参数

Table 1 Nano-scratch test parameters

\begin{tabular}{cc}
\hline Test parameters & Value \\
\hline Scratch crystal plane & $(100)$ \\
Scratch distance $/ \mu \mathrm{m}$ & 500 \\
Scratch speed $/\left(\mu \mathrm{m} \cdot \mathrm{s}^{-1}\right)$ & 5 \\
Load rate $\left./ \mathrm{mN} \cdot \mu \mathrm{m}^{-1}\right)$ & 0.25 \\
Normal load range $/ \mathrm{mN}$ & $0-125$ \\
Const normal load $/ \mathrm{mN}$ & $20 / 35 / 50$ \\
\hline
\end{tabular}

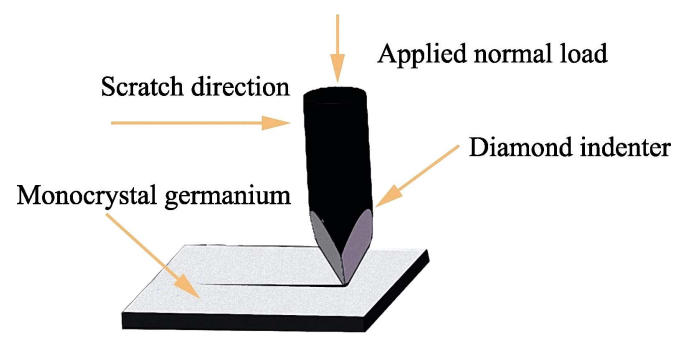

图 1 划刻实验示意图

Fig. 1 Diagram of nano-scratch experiment 

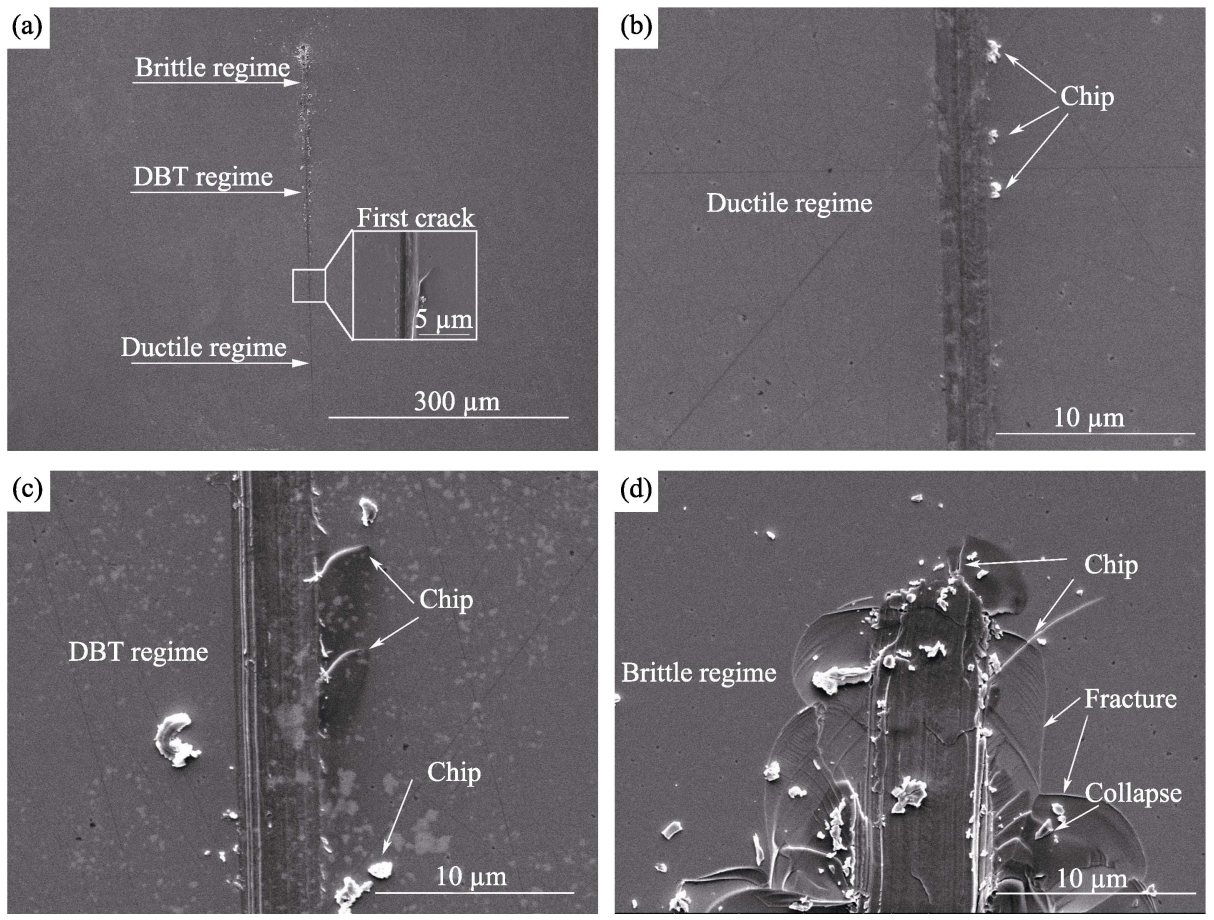

图 2 划痕的整体形貌及其局部放大图

Fig. 2 Scratch morphology and its local amplification

(a) Overall morphology; (b) Ductile regime; (c) DBT regime; (d) Brittle regime

当载荷增至脆塑转变临界深度时, 单晶锗工件 表面上开始出现裂纹, 以第一道裂纹萌生的位置作 为单晶锗发生脆朔转变的临界深度 $d_{\mathrm{c}}$, 由此进入到 脆塑转变域。图 2(c)为变载荷划刻后的划痕形貌特 征图, 在划刻距离为 $298 \mu \mathrm{m}$ 时发生脆塑转变, 其瞬 时划刻深度为 $469 \mathrm{~nm}$ 。

如图 2(d)所示, 在脆性域由于材料剧烈的断裂, 在工件表面造成壳状断裂与凹坑。根据划刻深度与 划刻距离间的关系, 将划刻域划分为延性域、脆塑 转变域与脆性域, 如图 3 所示。

\section{2 单晶锗划刻过程材料去除方式}

在划刻过程中，单晶锗将经过延性域（其中包 括弹性阶段与塑性阶段)、脆塑转变域及脆性域三个

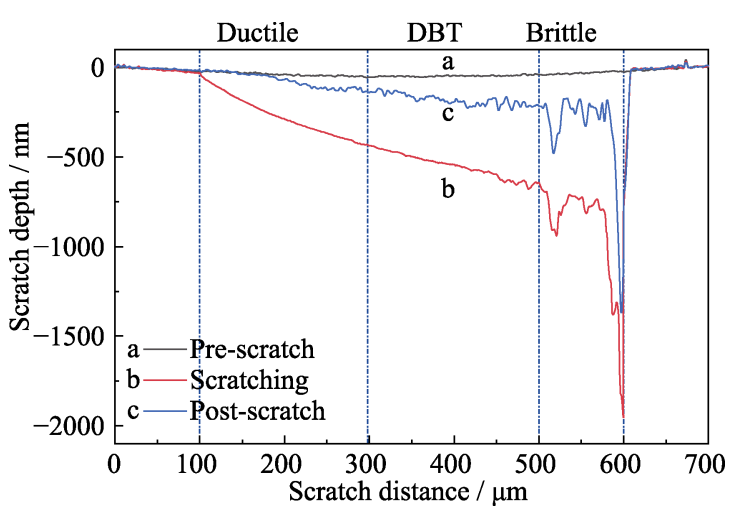

图 3 单晶锗划刻深度随划刻距离变化曲线

Fig. 3 Curves of scratch distance $v s$ depth for monocrystalline germanium
阶段。为了探明各个阶段单晶锗的材料去除机制, 辅以定载荷方式划刻, 工件表面的三维形貌如图 4。

在延性域划刻, 工件表面首先发生弹性变形, 不产生材料去除, 如图 4(a)所示。随着载荷的增加, 压头与工件接触区域发生塑性流动, 造成划痕边沿 的塑性隆起与褶皱, 并在沟槽两侧形成切屑, 如图 4(b) 所示。

根据断裂理论，当工件表面能与断裂能相等时， 工件表面开始产生脆性断裂 ${ }^{[8]}$ 。工件由此到脆塑转 变域, 单晶锗表面的塑性流动不断转化为脆性断裂, 并形成间歇性裂纹如图 4(c)。裂纹的分布情况如图 5 所示, 在塑性变形区域下, 由于压头的压应力产生 中心裂纹(Median crack); 同时工件受到拉、压与剪 切应力的共同作用 ${ }^{[9]}$, 在工件内部形成横向裂纹 (Lateral crack)。卸载时, 虽然中心裂纹由于工件的 弹性回复而闭合, 但横向裂纹会在残余应力的作用 下继续扩展。

在脆性域, 裂纹间相互扩展、延伸, 工件表面出 现严重的崩碎及片状剥落, 造成其加工精度和表面 完整性的下降，实际加工中需避免。不同划刻域的 载荷、划刻距离范围如表 2 所示。

\section{3 单晶锗定载荷划刻力学特征}

切削力不仅可以表征加工过程, 也反映加工状 态的主要参数, 切削力的大小直接影响工件的加工 状态及其表面质量 ${ }^{[10]}$ 。在纳米划刻实验中工件主要 
受切向力与法向力的影响, 其中切向力的主要来源 为工件克服弹、塑性变形抗力产生的摩擦力, 因此 有必要研究划刻过程中划刻力的变化趋势, 切向力 与法向力随划刻距离变化如图 6 所示。从划刻距离 为 $100 \mu \mathrm{m}$ 处载荷开始线性增加, 至 $600 \mu \mathrm{m}$ 处达到 最大载荷 $125 \mathrm{mN}$ 并完全卸载。

当工件处于非脆性域时, 幂函数很好地符合切

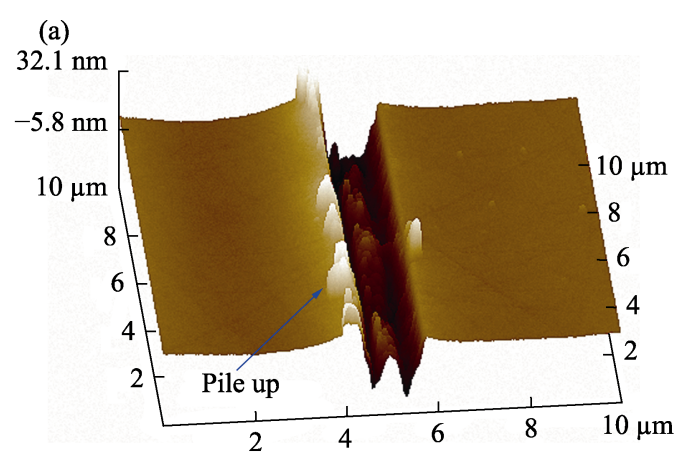

(b)

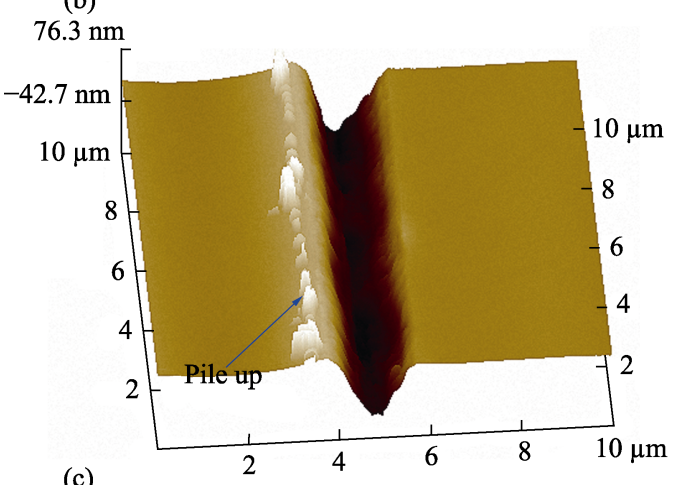

(c)

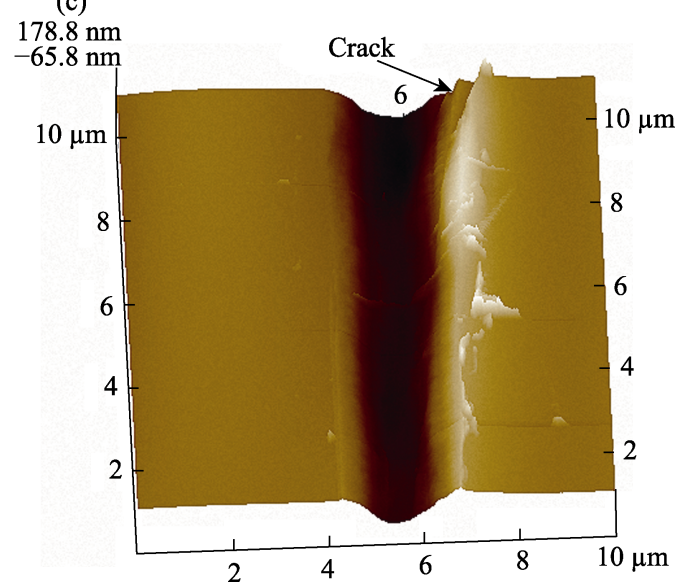

图 4 定载荷划刻的 AFM 图像

Fig. 4 AFM images of const load scratch (a) $20 \mathrm{mN}$; (b) $35 \mathrm{mN}$; (c) $50 \mathrm{mN}$

表 2 不同划刻域的载荷范围、划刻距离范围

Table 2 Load and scratch distance ranges for diffirent regimes

\begin{tabular}{ccc}
\hline Regime & Load range $/ \mathrm{mN}$ & Scratch distancerange $/ \mu \mathrm{m}$ \\
\hline Ductile regime & $0-48$ & $100-298$ \\
DBT regime & $48-100$ & $298-500$ \\
Brittle regime & $100-125$ & $500-600$ \\
\hline
\end{tabular}

向力 $F_{\mathrm{t}}$ 、法向力 $F_{\mathrm{n}}$ 随划刻深度的变化趋势, 采用最 小二乘法对其进行非线性拟合。当划刻深度继续增 至脆性域时，在单晶锗表面出现严重的断裂及大面 积的片状剥离, 使工件的切向力与法向力处于剧烈 的震荡中。本文对工件划刻全过程中 $F_{\mathrm{n}} 、 F_{\mathrm{t}}$ 随划刻 深度 $h_{v}$ 的变化进行了拟合, 如图 7, 拟合结果如式 (1) (4) 所示:

$$
\begin{gathered}
F_{\mathrm{n}}=6.984 \times 10^{-3} h_{v}{ }^{1.49}, h_{v} \in(0,636] \\
F_{\mathrm{t}}=3.968 \times 10^{-6} h_{v}{ }^{2.353}, h_{v} \in(0,636] \\
F_{\mathrm{n}}=-7.78 \times 10^{13} h_{v}{ }^{-4.655}+114, h_{v} \in(636,900] \\
F_{\mathrm{t}}=-8.61 \times 10^{5} h_{v}{ }^{-1.578}+47.6, h_{v} \in(636,900]
\end{gathered}
$$

其中式(1) (4)的相关系数 $r$ 分别为 $0.98 、 0.94 、 0.52$ 、 0.79 , 说明在非脆性域划刻力与压入深度存在强相 关关系。决定系数 $R^{2}$ 依次为 $0.997 、 0.996 、 0.057$ 、 $0.638, R^{2}$ 近似为 1 , 拟合函数很好地符合实验数据, 具有较高的可靠性。在脆性域时, 由于单晶锗表面 的严重断裂, $F_{\mathrm{n}}$ 与 $F_{\mathrm{t}}$ 剧烈波动, $R^{2}$ 显著低于 1 , 拟合 效果较差。结合本文 2.2 节, 虽然工件在脆塑转变时 会产生裂纹, 但力的波动并不明显, 在划刻过程中 塑性流动是能量消耗的主要方式。

\section{4 单晶锗划刻时弹性回复}

工件已加工表面的弹性回复会对刀具的后刀面

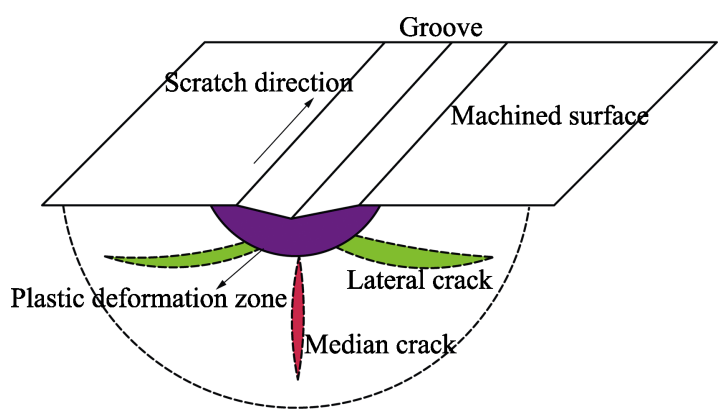

图 5 单晶锗裂纹分布示意图

Fig. 5 Diagram of crack distribution for monocrystalline germanium

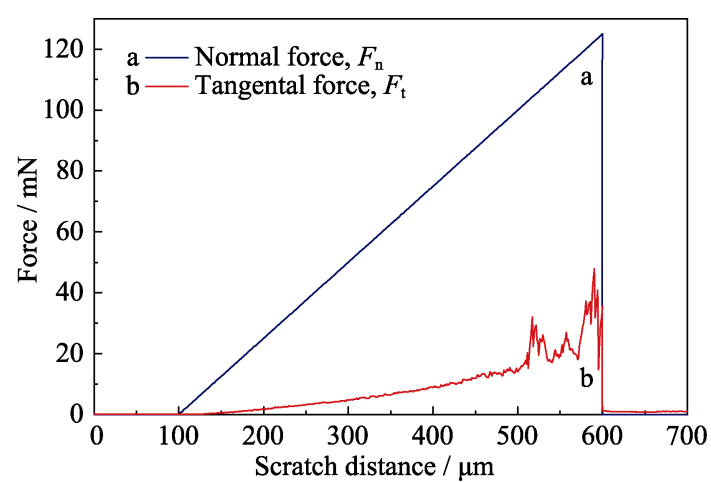

图 6 划刻力随划刻距离变化曲线

Fig. 6 Curves of scratch force vs distance 
产生摩擦 ${ }^{[1]}$, 影响其表面质量与面型精度。为满足 光学元器件对面型精度的要求, 需考虑在实际加工 过程中, 弹性回复对于切削的影响。

图 3 表明单晶锗划刻全过程中都存在材料的弹 性回复, 这会在压头和工件接触区域产生摩擦, 单 晶锗弹性回复率 $\eta$ 通过式(5)计算。

$$
\eta=\frac{h_{v}-h_{r}}{h_{v}} \cdot 100 \%
$$

其中 $h_{v} 、 h_{r}$ 分别表示单晶锗的压入深度和弹性回复 后的深度。

弹性回复率 $\eta$ 与划刻距离之间的关系如图 8, 从 100 至 $140 \mu \mathrm{m}$ 内此时最大载荷为 $10 \mathrm{mN}$, 单晶锗 工件表面的弹性回复率近似为 1 , 这时工件处于完
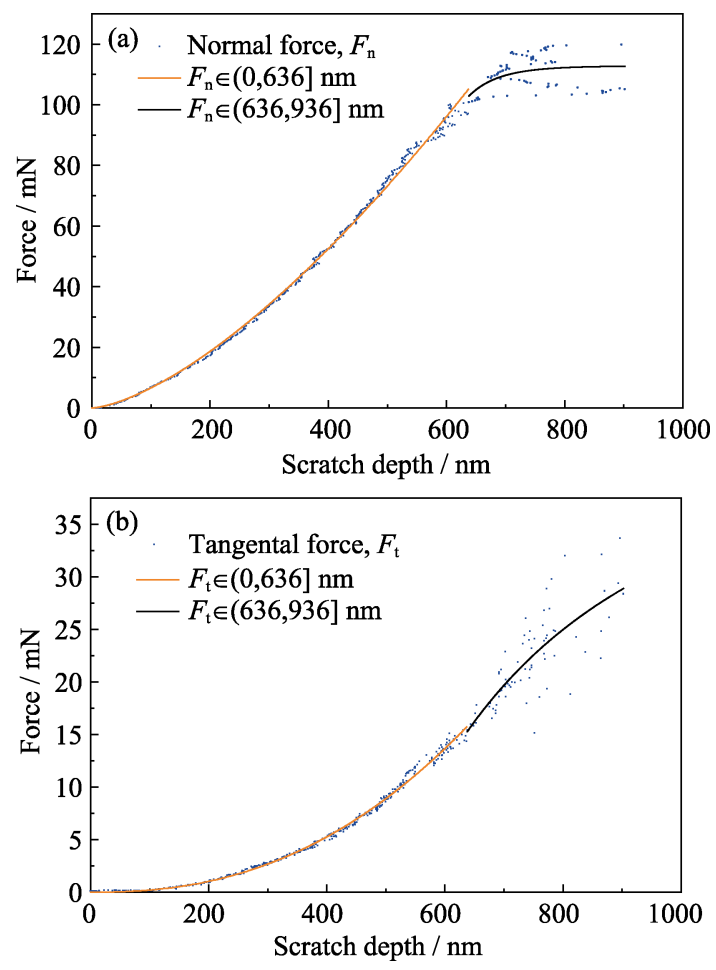

图 7 单晶锗划刻力随划刻深度变化曲线

Fig. 7 Curves of scratch force $v s$ depth for monocrystalline germanium

(a) Normal force; (b) Tangental force

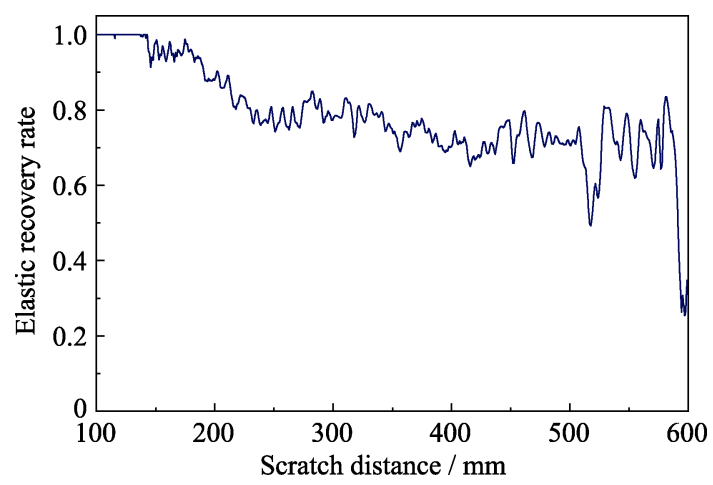

图 8 弹性回复率随划刻距离变化图

Fig. 8 Curve of elastic recovery rate during scratching
全弹性阶段。在其他区域内，由于载荷的增加，单晶 锗进入到弹塑变形阶段, $\eta$ 的值不会随着划刻距离 而大幅改变, 而是逐步下降到一个稳定值并小幅波 动，经计算 $\eta$ 在稳定阶段的平均值为 0.76 。

\section{5 单晶锗脆朔转变临界深度模型}

由于压头尖端曲率半径相对临界深度而言较小, 忽略其对压入深度 $h_{v}$ 的影响。当压入深度 $h_{v}$ 达到单 晶锗脆塑转变临界深度时, 将 Cube 压头视为理想 三棱雉, 压头几何形状如图 9, 其中心线与棱面夹 角 $\theta$ 为 $34.27^{\circ}$, 压头载荷 $P$ 与压入深度 $h_{v}$ 之间的关 系如式(6) ${ }^{[12-13]}$ 。

$$
P=\xi \cdot H \cdot A_{\mathrm{eff}}
$$

其中 $\zeta$ 表示压头几何因子, 本文取 $0.75, H$ 是单晶锗 显微硬度为 $9 \mathrm{GPa}$ 。对于雉形压头而言, 有效接触面 积 $A_{\mathrm{eff}}$ 为投影面积 $A_{\mathrm{p}}$ 的一半，则可得式(7)。

$$
P=\frac{1}{2} \xi \cdot H \cdot A_{\mathrm{p}}
$$

已有的研究往往忽视已加工表面的弹性回复 ${ }^{[12]}$, 这样使投影面积 $A_{\mathrm{p}}$ 的计算结果不够准确, 考虑到工 件的弹性回复, 根据划刻过程压头与工件接触模型 如图 9, 可通过式(8)计算 $A_{\mathrm{p}}$ 。

$$
A_{\mathrm{p}}=\sqrt{3}\left(2 h_{v}{ }^{2}+h_{v}\left(h_{v}-h_{r}\right)\right) \tan ^{2} \theta
$$

联立式(5)、(7)、(8)可得到在考虑弹性回复时压 头的载荷, 如式(9)所示。

$$
P=\frac{\sqrt{3}}{2}\left(2+\eta^{2}\right) \cdot \xi \cdot H \cdot h_{v}{ }^{2} \tan ^{2} \theta
$$

工件表面产生裂纹时存在临界载荷 $P_{\mathrm{c}}{ }^{[12]}$, 对于 Cube 压头而言, 当载荷 $P>P_{\mathrm{c}}$ 时, 会在工件内部形成 横向裂纹并扩展至表面; 当 $P<P_{\mathrm{c}}$, 则工件处于延性 域其表面光洁，临界载荷计算如式(10)。

$$
P_{\mathrm{c}}=\lambda \cdot \frac{K_{\mathrm{IC}}^{4}}{H^{3}}
$$

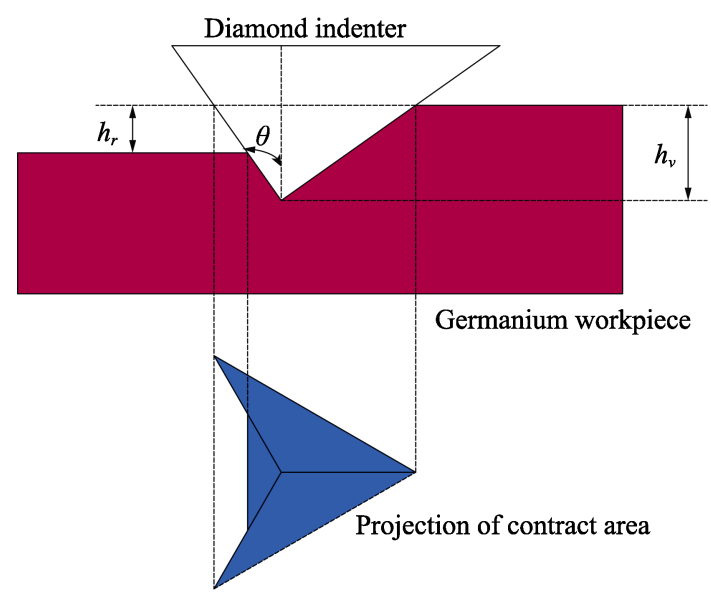

图 9 单晶锗划刻过程压头与工件接触模型

Fig. 9 Indenter-workpiece contract model in scratch process 
其中 $K_{\mathrm{IC}}$ 为单晶锗的断裂韧性, 取 $0.53 \mathrm{MPa} \cdot \mathrm{m}^{0.5[14]}$, $\lambda$ 为综合系数, 范围在 13500 20000 之间 ${ }^{[12]}$ 。联立式 (9) (10), 可得单晶锗脆塑转变临界深度 $d_{\mathrm{c}}$, 如式(11) 所示:

$$
d_{\mathrm{c}}=\sqrt{\frac{2 \lambda}{\sqrt{3}\left(2+\eta^{2}\right) \zeta \tan ^{2} \theta}}\left(\frac{K_{\mathrm{IC}}}{H}\right)^{2}
$$

根据本文实验结果在单晶锗弹性回复率 $\eta$ 约为 0.76 , 将脆塑转变临界深度为 $498 \mathrm{~nm}$ 带入式(11), 求得适用于单晶锗的综合系数 $\lambda$ 为 16029.5 。

\section{3 结论}

1) 根据单晶锗划刻后的表面形貌特征将划刻 域分为延性域、脆塑转变域与脆性域三个阶段, 并 对各个阶段的材料去除方式进行分析。

2) 研究了划刻全过程的弹性回复, 发现单晶 锗在纯弹性阶段的弹性回复率接近 1, 在塑性域与 脆塑转变域其弹性回复率约为 0.76 。

3) 拟合了划刻过程中切向力、法向力随划刻深 度变化函数, 利用相关系数进行检验, 结果表明在 延性域划刻力与划刻深度之间存在强相关性。

4) 基于纳米划刻实验, 首次结合已加工表面 的弹性回复, 提出一种适用于计算单晶锗脆塑转变 临界深度模型, 对于指导单晶锗超精密加工中切削 参数的选取、提高工件表面质量具有重要意义。

\section{参考文献:}

[1] XIE QI-MING, LI MAO-ZHONG, CHEN JUN-QI, et al. The research on technology of two-denmension polygon Ge crystal by single point diamond fly-cutting. New Technology \& New Process, 2009, 3: 22-24.

[2] YAN J, MAEKAWA K, TAMAKI J, et al. Experimental study on the ultraprecision ductile machinability of single-crystal Germanium. JSME International Journal Series C, 2004, 47(1): 29-36.
[3] SUZUKI N, YAN Z, HINO R, et al. Ultraprecision Micromachining of Single Crystal Germanium by Applying Elliptical Vibratlon Cutting. Nagoya: Jspe Semestrial Meeting, 2006: 545- 546.

[4] WANG J, FANG F, ZHANG X. An experimental study of cutting performance on monocrystalline germanium after ion implantation. Precision Engineering, 2015, 39: 220-223.

[5] RAVINDRA D, GHANTASALA M K, PATTEN J. Ductile mode material removal and high-pressure phase transformation in silicon during micro-laser assisted machining. Precision Engineering, 2012, 36(2): 364-367.

[6] LI C, ZHANG F, MENG B, et al. Research of material removal and deformation mechanism for single crystal GGG $\left(\mathrm{Gd}_{3} \mathrm{Ga}_{5} \mathrm{O}_{12}\right)$ based on varied-depth nanoscratch testing. Materials \& Design, 2017, 125: 180-188.

[7] ZHANG L, TANAKA H. Towards a deeper understanding of wear and friction on the atomic scale - a molecular dynamics analysis. Wear, 1997, 211(1): 44-53.

[8] CHOI D, LEE J, KANG N, et al. Study on ductile mode machining of single-crystal silicon by mechanical machining. International Journal of Machine Tools and Manufacture, 2017, 113: 1-9.

[9] DENG CHAO-HUI, ZHANG BI, SUN ZHONG-YU, et al. The material removal mechanism of ceramics in grinding process. Diamond \& Abrasives Engineering, 2002, 2: 47-51.

[10] ZHANG CHENG-LONG, FEN PIN-FA, WU ZHI-JUN, et al. Mathematical modeling and experimental research for cutting force in rotary ultrasonic drilling. Journal of Mechanical Engineering, 2011, 15: 149-155.

[11] ARIF M, XINQUAN Z, RAHMAN M, et al. A predictive model of the critical undeformed chip thickness for ductile brittle transition in nano-machining of brittle materials. International Journal of Machine Tools and Manufacture, 2013, 64: 114-122.

[12] MA L, GONG Y, CHEN X. Study on surface roughness model and surface forming mechanism of ceramics in quick point grinding. International Journal of Machine Tools and Manufacture, 2014, 77: 82-92.

[13] ZONG W J, CHENG K, LI D, et al. The ultimate sharpness of singlecrystal diamond cutting tools-Part I: Theoretical analyses and predictions. International Journal of Machine Tools and Manufacture, 2007, 47(5): 852-863.

[14] LEMAITRE P. Fracture toughness of germanium determined with the Vickers indentation technique. Journal of Materials Science Letters, 1988, 7(8): 895-896. 